\title{
When unsupervised training benefits category learning
}

\author{
Franziska Bröker ${ }^{1,2, \varpi}$, Bradley C. Love ${ }^{3,}$, and Peter Dayan ${ }^{1,4,{ }^{*}}$ \\ ${ }^{1}$ Max Planck Institute for Biological Cybernetics, Tübingen, Germany \\ ${ }^{2}$ Gatsby Computational Neuroscience Unit, London, UK \\ ${ }^{3}$ Experimental Psychology, University College London, London, UK \\ ${ }^{4}$ University of Tübingen, Tübingen, Germany \\ ${ }^{\bowtie}$ Corresponding author: Franziska Bröker, franziska.broeker@tuebingen.mpg.de \\ *equal contribution
}

Humans continuously categorise inputs, but only rarely receive explicit feedback as to whether or not they are correct. This implies that they may be integrating unsupervised information together with their sparse supervised data - a form of semi-supervised learning. However, experiments testing semi-supervised learning are rare, and are bedevilled with conflicting results about whether the unsupervised information affords any benefit. Here, we suggest that one important factor that has been paid insufficient attention is the alignment between subjects' internal representations of the stimulus material and the experimenter-defined representations that determine success in the tasks. Subjects' representations are shaped by prior biases and experience, and unsupervised learning can only be successful if the alignment suffices. Otherwise, unsupervised learning might harmfully strengthen incorrect assumptions. To test this hypothesis, we conducted an experiment in which subjects initially categorise items along a salient, but task-irrelevant, dimension, and only recover the correct categories when sufficient feedback draws their attention to the subtle, task-relevant, stimulus dimensions. By withdrawing feedback at different stages along this learning curve, we tested whether unsupervised learning improves or worsens performance when internal stimulus representations and task are sufficiently or insufficiently aligned, respectively. Our results demonstrate that unsupervised learning can indeed have opposing effects on subjects' learning. We also discuss factors limiting the degree to which such effects can be predicted from momentary performance. Our work implies that predicting and understanding human category learning in particular tasks requires assessment and consideration of the representational spaces that subjects entertain for the materials involved in those tasks. These considerations not only apply to studies in the lab, but could also help improve the design of tutoring systems and instruction.

Keywords: semi-supervised learning | categorisation | representation 


\section{Introduction}

Humans learn to categorise both with and without explicit feedback or supervision. For example, children learn to tell things apart, like a sheep from a goat, with the help of at least some explicit labels provided by adults. However, the adults rather rarely receive this kind of explicit feedback on many of their continuing categorisations. Even though a hybrid of supervision and mere exposure appears to characterise human learning well, studies in the laboratory have largely, and rather artificially, separated the forms of learning. To understand how human learning works under more natural conditions, we need to understand the joint contributions and interactions of both supervision and exposure.

Recently, various experiments have examined semi-supervised learning in adults. However, results conflict. One line of research on semi-supervised categorisation shows that humans adjust the positions of one-dimensional category boundaries acquired with supervision when presented with a swathe of unsupervised samples whose distribution suggests a slightly shifted boundary [Zhu et al., 2007, Lake and McClelland, 2011, Kalish et al., 2012, 2015, Gibson et al., 2015]. These results provide evidence that humans indeed integrate supervised and unsupervised information.

By contrast, work that investigated whether subjects' categorisation performance improved when intermixing supervised with unsupervised training trials in two-dimensional tasks did not find conclusive evidence: Unsupervised trials have been reported to have no effect on categorisation accuracy in tasks that require subjects' to integrate information from only one (rule-based) or multiple stimulus dimensions (information integration) [Vandist et al., 2009, McDonnell et al., 2012], or only to affect response speed [Vandist et al., 2019], or only to benefit learning under time pressure [Rogers et al., 2010], or only to boost generalisation performance in relational category learning if unsupervised and supervised stimuli are similar [Patterson and Kurtz, 2018].

These results show that while the idea that humans should be able to boost supervised information by integrating it with unsupervised information appears convincing, the literature only provides sparse and conflicting evidence, corrupted by substantial differences in experimental designs (e.g., tasks, presentation times, response requirements). In fact, the mixed results have been taken as evidence that semi-supervised learning may only be beneficial under limited conditions (e.g., under time pressure or late in learning; Vandist et al. [2019]) and that perhaps supervised items enjoy a special status, which is why they appear to be weighed more strongly in learning [McDonnell et al., 2012, Lake and McClelland, 2011, Vandist et al., 2009, Zhu et al., 2010].

One way to understand these conflicting results is to consider category learning experiments from a more abstract viewpoint. An experimenter defines a task by specifying a mapping from a set of stimuli to a set of labels. The set of stim- 
uli is typically constructed by specifying dimensions of variations in a collection of features. Some, or all, of the feature values of a stimulus, i.e., its coordinates along these feature dimensions, then systematically map onto a category label. The subject needs to learn this mapping, and thus also at least something about the feature dimensions that differentiate the categories. However, the subject can only operate on an internal representation of stimuli, that is on their coordinates along internal feature dimensions. These dimensions will have been shaped by the subject's prior experience. Thus, successful learning involves three components: (i) adjustment or augmentation of internal representations so they align sufficiently with the dimensions critical for the experimenter's categorisation (e.g. by attending to task-relevant dimensions while suppressing others, or by learning appropriate feature representations for a given task); (ii) separation of stimuli into categories given these representations (i.e., the explicit or implicit learning of category boundaries); and (iii) assignment of the correct labels to these categories.

Although supervised learning supports all three aspects of the acquisition of categories, unsupervised learning does not. Learning the correct label for a class, by definition, requires supervision. However, and more importantly, unsupervised learning is limited to tailoring representations and learning category boundaries from distributional information available in the representation evoked by the presented stimuli. Thus, we hypothesise that unsupervised learning can only find and refine categories that are somewhat obvious given existing representations (e.g. obvious groups or spectral structure). By contrast, supervision will be necessary to induce gross changes to representations if they are misaligned with the task. This could involve shifting attention to subtle feature dimensions, or acquiring new such dimensions.

In sum, learning will be constrained by existing representations, especially in the absence of feedback (Fig. 1). For instance, subjects may simply not attend to a task-relevant stimulus dimension, in which case distributional information available in that dimension will be inaccessible for learning [Rogers et al., 2010, Gibson et al., 2013]. This is equivalent to subjects' more obvious inability to learn seemingly random stimulus-category assignments without supervision. More subtly, even the ability to integrate information from new and old stimuli to refine categorisations will be governed by the representations prevailing at the time the old stimuli were presented. This is because these representations constrain what aspects of those stimuli will have been retained. 
(a)

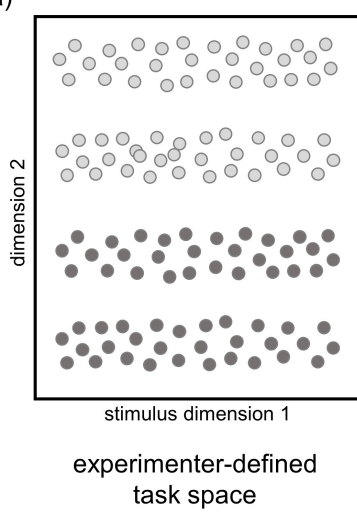

(b)

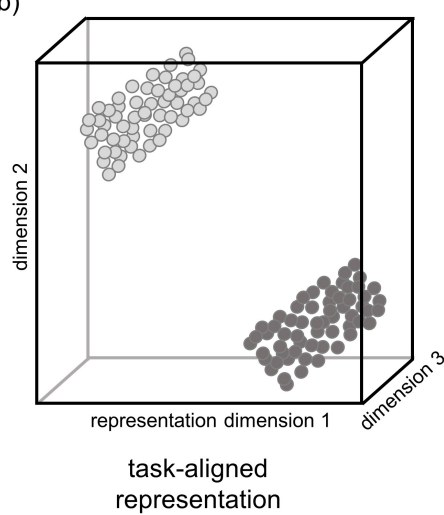

(c)

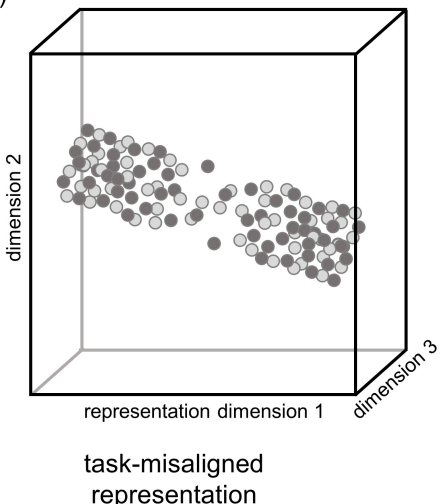

Figure 1: Schematic illustration of a task structure and examples of task-aligned representations and task-misaligned representations for which unsupervised learning would help and hurt learning in the task respectively. (a) An experimental task in which stimuli are drawn from two categories (shown by dark and light grey circles) that extend along two pre-defined dimensions (e.g. the length and orientation of lines). (b) Task-aligned representations entertained by learners neither need to retain all information about the original input, nor do they need to be perfectly parallel to the experimenter-defined dimensions. It suffices for stimuli of the same category to be represented similarly, thus supporting correct responses in this particular task even in the absence of feedback. We hypothesise that, in this case, learners' performance can improve without supervision by continued representational learning (e.g. further within-category compression). (c) A representation is task-misaligned if stimuli from the same category are not well separated. We hypothesise that unsupervised learning cannot improve performance if representations are misaligned because unsupervised information can only strengthen wrong assumptions about the underlying category structure. It is only with sufficient supervision that misaligned representations can be corrected, enabling future unsupervised learning to be useful.

To illustrate these points, consider a young child learning that some animals are called fish while others are called mammals. By exploiting an internal representation which reports that fish possess the feature of living in water, the child may quickly learn to distinguish mammals from fish. This allows them to categorise new types of fish without feedback, whilst at the same time learning about the various appearances of fish and their distribution. However, this simple clustering is misaligned with the true category of fish, because exceptions, such as whales, live in water but are mammals. The child will be unable to perfect their fish categorisation unless they receive sufficient explicit feedback that allows them to learn to represent aquatic mammals differently from fish. By contrast, consider another child who has 
more experience with these two categories or learned to represent them separately more quickly because of frequent visits to aquaria. This child might, in the same situation, be able to learn from additional observations alone by fine-tuning existing clusters of these aquatic animals.

Accordingly, unsupervised trials can only improve performance in an experiment if subjects' representational space suggests a similarity structure between stimuli that is sufficiently aligned with the experimenter-defined category structure so that simple refinements suffice. We call this the semi-supervised representation-to-taskalignment hypothesis. This hypothesis is consistent with Hammer's (2015) suggestion that feature saliency of stimuli should influence the degree to which visual category learning is dependent on feedback as well as Rogers et al.'s (2010) suggestion that attentional mechanisms influence the availability to the learner of relevant distributional information.

Returning to the observation of conflicting results in semi-supervised learning experiments, it is important to consider that internal representations are shaped by prior experience, which differs substantially between individuals. Thus, unsupervised learning may have very different, and even opposite, effects on individuals even within exactly the same task. In addition, the modest amounts of supervision provided in these experiments may have a greater effect on the representations of some subjects than others, either because of intrinsic differences or because of prior learning or meta-learning. This may also lead to differential effects of subsequent unsupervised exposure in the same task.

In the context of learning in the real-world, this would suggest that people with different experience and different rates of learning can either be helped or hurt by unsupervised learning in a specific task because their representations differ. For the example of learning to distinguish sheep from goats, we can assume that the vast majority of readers are non-experts in this domain, yet have encountered many exemplars (mostly unsupervised) over the course of their life and probably have learned about some of the characteristics that are typical for these animals which determine their internal representations. One of these salient features is that sheep are typically woolly whereas goats are not. But few non-experts may be aware that breeds of sheep and goats exist that are indistinguishable based on woolliness, or other apparently salient characteristics. Instead, experts (but surprisingly few nonexperts) rely on a much simpler, discrete and diagnostic feature to categorise sheep and goats: whether their tail points up (goats) or down (sheep). That is, despite this simple-to-learn feature and repeated supervised and unsupervised exposure over a lifetime, many non-experts confidently categorise with respect to an irrelevant, but highly salient and often sufficient feature (e.g. woolliness), apparently failing to include the true discriminating feature in their representations. As a result, non-experts correctly categorise only those animals who conform to their incorrect perception (woolly sheep) but continue to make mistakes on rare instances (woolly goats). This example illustrates that exposure alone does not automatically lead to 
the accurate discovery of categories, but that non-experts may instead practice the wrong categorisation in the absence of feedback because their representations impose a view on the inputs that is misaligned with the task and not trivially revised.

In this paper, we emulate this real-world learning problem to test our semisupervised representation-to-task alignment hypothesis. This hypothesis entails that unsupervised learning can have opposite effects on individuals' performance within the exact same category learning task depending on the representations they entertain. We test this by creating a learning task in which unsupervised trials can help or hurt the performance of individual subjects depending on the degree to which their internal representation and experimenter-defined task are aligned at the outset of unsupervised training. Since we cannot assess the representations different subjects entertain directly, we infer them from subjects' momentary categorisation responses.

We first describe this experimental design in detail. We then present our results which provide evidence that unsupervised learning can indeed have seemingly opposing effects on subjects' behaviour even within a single behavioural task consistent with the semi-supervised representation-to-task alignment hypothesis. We conclude by discussing the limitations and difficulties of assessing and predicting such effects in categorisation experiments in general and elaborate on possible mechanisms underlying semi-supervised learning. 


\section{Experiments}

In order to test the semi-supervised representation-to-task alignment hypothesis, we designed a simple categorisation task in which we could measure and manipulate the degree of alignment between a subject's internal representational space and our experimenter-defined task space. We measured alignment by evaluating subjects' responses against two category boundaries (one correct, one incorrect) as they learned; and we manipulated the degree of alignment by withdrawing supervision at different stages of subjects' learning.

We adapted the task of Ramscar et al. [2010] to mimic the problem of learning to distinguish sheep from goats described above. That is, our stimulus material was designed such that, in the absence of corrective feedback, most subjects spontaneously categorised stimuli along a salient continuous dimension that was task-irrelevant (the equivalent of woolliness) and whose uniform distribution did not favour a class distinction. However, we also provided simple, discrete, features (the equivalents of the tail orientation) that were rarely noticed spontaneously, but actually defined a group structure. Given sufficient feedback, subjects could learn to assign stimulus groups to categories correctly by relying on these subtle features. We expected that further unsupervised learning would only be beneficial when subjects were attending appropriately to these features (Fig. 2).
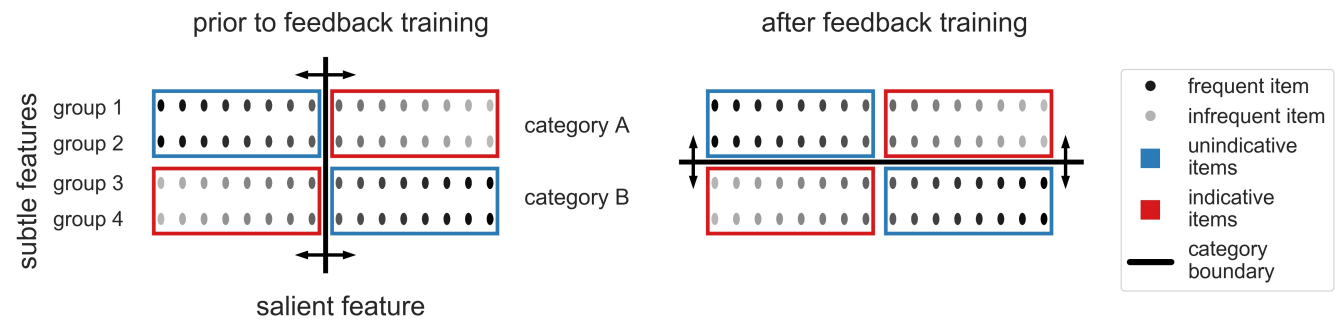

Figure 2: Schematic illustration of the stimulus and task structure. Stimuli vary according to different features. One stimulus feature is continuous and salient (but task-irrelevant) such that subjects naturally perceive similarity and categorise stimuli along this dimension in the absence of feedback. The other stimulus features are more subtle and indicate membership to four underlying groups which themselves are assigned to two categories that define the task subjects are asked to learn. In such a task, we expect that subjects will need feedback in order to learn to categorise items in accordance with the task. The task includes two types of stimuli: those that should be categorised correctly independent of the correct or incorrect representational structure (unindicative stimuli) and those that reflect the underlying representation (indicative stimuli). Crucially, stimuli are sampled such that the distribution over the salient dimension is uniform, while the subtle dimensions defines discrete groups. 
In order to test our hypothesis, we assessed the effect of feedback withdrawal after an initial supervised phase. We predicted that subjects' internal representation at this point of withdrawal would affect whether further unsupervised exposure would help or hurt performance. If feedback is withdrawn when a subject still entertains a task-inappropriate representation, either because they adapted slowly in response to the feedback or because their prior representations were particularly inappropriate, then unsupervised learning would hurt performance. On the other hand, if feedback is withdrawn when a subject already entertains a task-appropriate representation (because their representation was more aligned from the start or they adapted quickly in response to the feedback), they would be able to improve by learning from exposure alone.

Several methodological considerations shaped the details of the experimental design: firstly, the question of how to assess representations; secondly, how to evoke representational change; and lastly how to chose the time point of feedback withdrawal. We will now explain how we addressed these three questions and how our solutions result in the qualitative predictions of learning curves displayed in Fig. 3.

The most important methodological prerequisite for this study is the ability to draw conclusions about subjects' internal representations. We achieved this indirectly by evaluating performance on two classes of stimuli: frequently occurring unindicative stimuli that subjects would assign to the correct category irrespective of whether they attended to the task-relevant or task-irrelevant dimension of the stimuli (the equivalent of woolly sheep; thus performance on these stimuli has no implications for subjects' internal representation), and less frequent, indicative stimuli that would either be categorised correctly or incorrectly (the equivalent of woolly goats; performance on these indicates whether subjects attended to the correct dimension). This design allows us to make qualitative predictions about subjects' performance depending on which representation they entertain, and thus make inferences about subjects' representations from their performance.

Another methodological prerequisite was to ensure subjects underwent representational change in learning to solve the task. That is, before being able to test our semi-supervised representation-to-task-alignment hypothesis by withdrawing feedback at different stages of learning, we first needed to ensure that subjects' learning under fully unsupervised and fully supervised baseline conditions followed the precepts laid out above. That is, we first needed to establish that subjects would initially categorise according to the task-irrelevant feature (i.e., woolliness, by analogy) but could learn to categorise by the task-relevant feature (i.e., the tail, by analogy) when receiving feedback. As we will see below, we were particularly interested in the possibility of substantial individual differences in learning trajectories so that withdrawing feedback at a single, pre-determined point during acquisition would amount, across the whole group of participants, to withdrawing it at quite different states of representational alignment. 
For this purpose, we conducted two calibration studies to ensure that the experimental parameters produced the desired learning curves under baseline conditions. First, we tested whether subjects would indeed perceive similarity between stimuli along the wrong, continuous dimension initially. This would be reflected in an improvement on the unindicative items, but diminishing performance on indicative items in the absence of corrective feedback (unsupervised learning) as subjects strengthen their incorrect beliefs about category structure (Fig. 3 a).

Having confirmed that subjects were not sensitive to the experimenter-defined group structure, but rather categorised items along the salient continuous dimension, the second calibration study tested subjects while providing feedback throughout (supervised learning) so that subjects were all expected to learn the experimenterdefined category structure. In this case, an improvement on both unindicative and indicative items would be expected. However, performance on indicative items would be predicted to increase more slowly because task-relevant and task-irrelevant features suggest different responses, and thus subjects might only gradually recover from an initially incongruous representation. In particular, since subjects learn at different rates, we predicted that the performance of some learners would approach perfection monotonically and quickly (fast learners), but, crucially, that others would exhibit a U-shaped learning curve in which they initially endorse the wrong category representation before they accumulate sufficient error and eventually recover the task-relevant representation (slow learners). These predictions are based on some of the principles suggested to underlie the famous U-shaped learning curves in language acquisition. Indeed, our experiment may serve as a categorisation analogue of these (Fig. 3 b, c).

Having established subjects' learning curves under fully unsupervised and supervised training in the calibration studies, we needed to decide on the time point of feedback withdrawal in order to test our hypothesis that representational alignment mattered. Since we do not assess representations and their alignments to the task directly, but rather infer them from noisy performance measures on the different item types, we exploited the fact established in our calibration studies that subjects learn at different rates. Thus, we could remove feedback after a subject-independent number of trials, and still access a suitable distribution of implied representational alignment. Concretely, we predicted that fast learners, who had grasped the task at the time of feedback withdrawal, would successfully self-improve on both indicative and unindicative items reaching similar performance to that under full supervision (Fig. 3 d). By contrast, we predicted that slow learners, who still attended to the wrong stimulus dimension at the time of feedback withdrawal, would improve only on the unindicative items but would worsen on indicative items, with performance approaching that of fully unsupervised learning (Fig. 3 e). Of course, the fact that we cannot assess representations directly will still limit our capacity to predict unsupervised effects on an individual subject basis. However, the predicted effects should be expressed on a group-level: The subjects that benefit from unsupervised 
trials are expected to have higher average performance before feedback withdrawal than the subjects that worsened in the face of unsupervised trials.
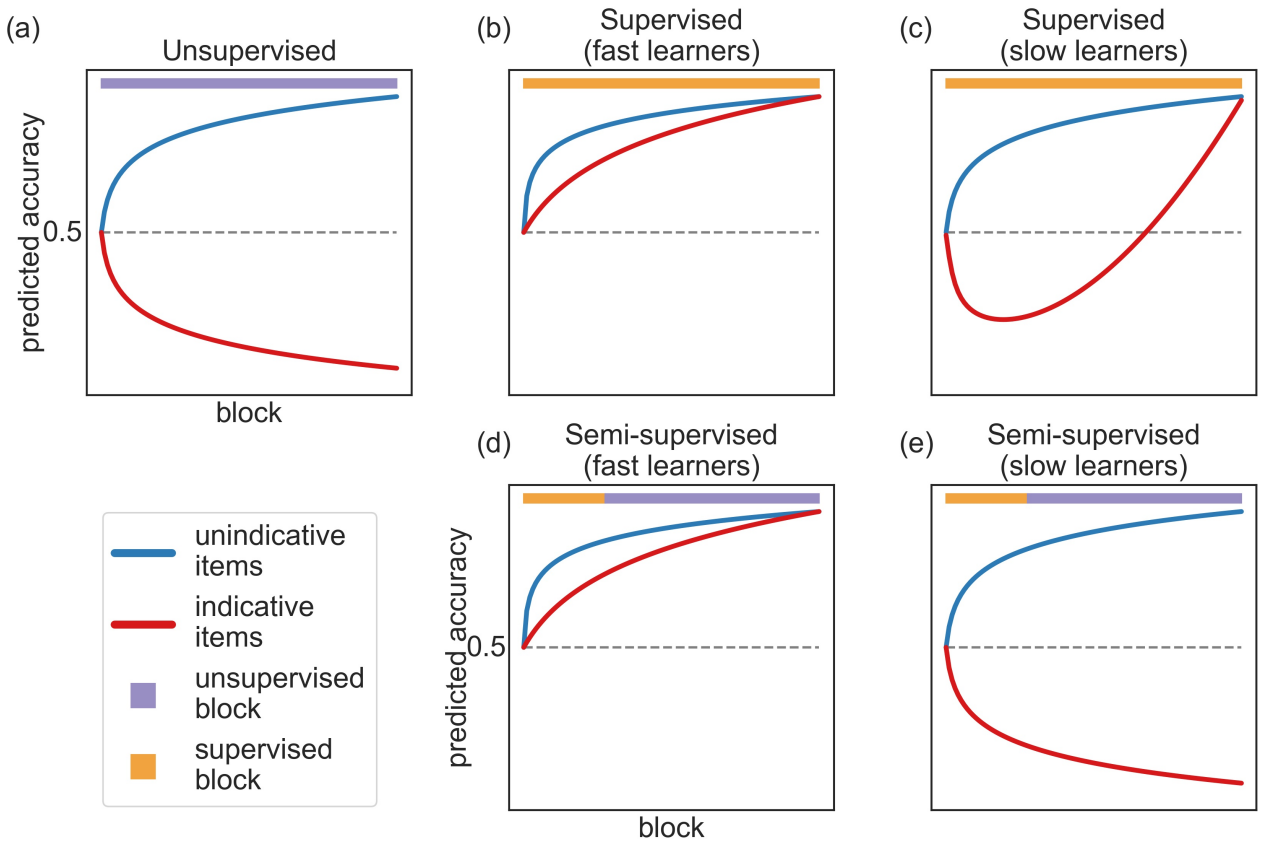

(e) Semi-supervised

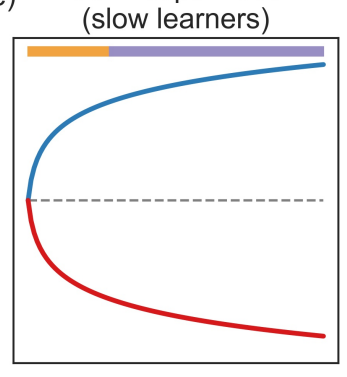

Figure 3: Predicted learning curves under different feedback conditions. Subjects are expected to reach high performance on unindicative items irrespective of feedback condition since performance is independent of whether subjects attend to the task-relevant or task-irrelevant feature. (a) Unsupervised condition: In the absence of feedback, subjects are expected to categorise by the task-irrelevant dimension and thus perform poorly on indicative items (including the appropriate remapping of responses to account for the fact that it is impossible for subjects to know which label is attached to which category in the absence of feedback). (b, c) Supervised condition: In the presence of feedback, subjects are expected to improve on indicative items as they learn to categorise by the task-relevant stimulus features. Fast learners are expected to approach perfection quickly. By contrast, slow learners are expected to recover from their initial reliance on the task-irrelevant dimension more slowly, hence displaying a U-shaped learning curve. (d, e) Semi-supervised condition: Subjects are expected to perform well on unindicative items at the time of feedback withdrawal and to further improve even in the absence of feedback. However, the benefit of unsupervised trials for indicative items is expected to depend on proficiency at the point that feedback is withdrawn: fast, proficient learners are expected to improve, while slow, inaccurate learners are expected to worsen. 


\subsection{Materials and Methods}

\subsubsection{Participants}

A total of 464 participants completed the calibration studies and the semi-supervised experiment via Amazon Mechanical Turk. 19 subjects completed the unsupervised calibration study for which they were paid $\$ 1.20$ irrespective of their performance and 14 of them entered analyses after exclusion (see below). Subjects who completed the supervised calibration study and semi-supervised experiment (232 and 213 subjects respectively) received $\$ 1.50$ irrespective of their performance. After exclusion 157 subjects entered the analysis of supervised training and 156 subjects that of semi-supervised training.

\subsubsection{Stimuli}

We sought to design visual stimuli that would initially attract subjects attention to a salient but task-irrelevant feature that varied continuously between two visually distinct shapes suggesting two plausible (but incorrect) categories to the observers. In addition, stimuli needed to have more subtle, discrete features that would be diagnostic of category membership, but that required supervision to learn that they were indeed task-relevant.

To achieve this, the composition of these features into artificial objects was inspired by the fribbles created by Michael Tarr and colleagues [Williams, 1998]. All four stimulus groups shared the same salient body dimension which morphed continuously between two distinctive shapes. Groups differed in four subtle appendages attached to the body which were all equally (and deterministically) diagnostic of the four groups that underlay the two categories. In addition, each appendage had three possible realisations varying slightly in colour, texture and shape (Fig. 4 a). Exemplars of four artificial object families were created using Blender 2.8 and images displayed to participants at a fixed size of $500 \times 889 \mathrm{px}$. Unsupervised and supervised pilot studies were conducted to optimise visual properties and number of the clusters in order to obtain the distinctive learning curves under both baseline conditions. 
(a)

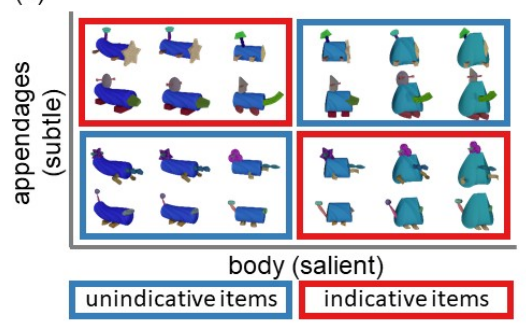

(b)

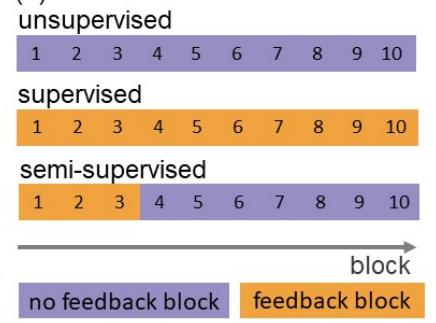

(c)

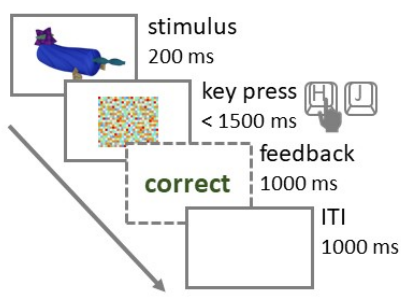

Figure 4: Experimental design. (a) Examples of stimuli from each of the four groups varying in a salient continuous feature and subtle discrete features. The distribution of the continuous feature was uniform when averaged across groups. (b) Number of feedback and no feedback blocks for the three experimental conditions. (c) In each trial, participants first saw the stimulus for $200 \mathrm{~ms}$, after which a mask appeared. Subjects indicated their response by pressing one of two pre-allocated keys on their keyboard. If they failed to respond within $1500 \mathrm{~ms}$, a response timeout message was provided as an instruction to respond faster next time. Upon their response in supervised, but not unsupervised trials, subjects received corrective feedback for $1000 \mathrm{~ms}$. The next trial started after a $1000 \mathrm{~ms}$ ITI. To maintain the same rhythm of responding in the unsupervised phase of the semi-supervised condition, the ITI was adjusted to $2000 \mathrm{~ms}$ after feedback withdrawal.

\subsubsection{Task Design}

All participants underwent 10 blocks comprising 16 trials each in which they had to categorise items into two categories (for some participants in the semi-supervised experiment three additional supervised blocks were appended at the end of the experiment for an exploratory analysis, but these data are not reported here). The experiment lasted between 8-12 minutes depending on feedback condition and, once started, subjects were not able to pause. Each block constituted of a pseudo-random arrangement of exemplars covering 16 equally spaced points on the body morph continuum and a random selection of appendage variations. Stimuli were sampled from a triangular proposal distribution such that more extreme body shapes were more likely to occur for unindicative stimuli while the overall probability of body shapes remained uniform across all stimulus groups. The sampling process was constrained to yield 4 members of each stimulus group as well as 6 indicative and 10 unindicative stimuli in every block. To achieve a balanced number of indicative and unindicative stimuli for each stimulus group across blocks, every other block contained 2 (un-)indicative stimuli from group 1 and 3, but 1 indicative and 3 unindicative stimuli from group 2 and 4 (and vice versa on the interleaving blocks). Two pseudo-random stimulus lists were sampled in this way and two additional lists constructed by reversing them. Subjects were randomly assigned to one of those four lists. 
Subjects in the unsupervised calibration study were allowed to choose the response key mapping themselves and were simply instructed to categorise objects into two classes depending on their perceived similarity. In the supervised calibration study subjects were instructed to learn the categories with the help of corrective feedback. While in the semi-supervised experiment, they were told in addition that after an unknown time, feedback would no longer be provided and that they should try to continue to categorise as best as they can.

On each trial, participants pressed one of two keys to indicate the category of the presented item (Fig. 4 c). Subjects in the supervised calibration study, but not the unsupervised calibration study, received corrective feedback. Based on the results from these studies, subjects in the semi-supervised experiment received feedback only for the first three blocks after which feedback was omitted for the rest of the experiment (Fig. 4 b).

All functionality of the experiment was implemented in custom-written JavaScript.

\section{Exclusion Criteria}

Participation in the studies was restricted to devices with a minimal screen size of $15.9 \times 31.8 \mathrm{~cm}$ to ensure images displayed in the desired size and to prevent the use of mobile devices. In addition, we excluded participants who either reported technical issues during the experiment, had incomplete data due to recording issues, or displayed behaviour suggesting they were not focussing on the task. Throughout the task, performance was tracked to ensure that participants were attentive. Performance warnings were presented on the screen for 10 seconds if a subject either responded too fast (less than $300 \mathrm{~ms}$ ), too slowly (more than $1500 \mathrm{~ms}$ ) or with an invalid key more than 6 times within the last 12 trials. Subjects that received more than two such performance warnings were excluded from the analysis, as well as subjects that uninterruptedly pressed the same or alternated keys for 12 trials (supervised training) or, in the case where responses were less regulated by feedback, 18 trials (unsupervised, semi-supervised training).

\subsection{Results and Discussion}

Throughout our analyses, reported response accuracies treat all missing or invalid responses as incorrect trials. As explained above, we had designed the experiment such that we could infer which representation subjects were likely to entertain at different times in the experiment (and hence infer their alignment with the task) from subjects' momentary performance on indicative and unindicative items. We used this to define which subjects had likely learned the appropriate representation aligned with the task. For that, we applied a fixed performance criterion of above $60 \%$ accuracy on each items type (indicative as well as unindicative) averaged over the last four blocks of the experiment to conclude that subjects had learned the underlying task. We call this performance criterion the conformant-categorisation 
criterion and subjects that reached it conformant learners for their behaviour conforms to what is expected from noisy learners that entertain the task-appropriate representation. We applied a fixed performance criterion of above $60 \%$ accuracy on unindicative and below $40 \%$ on indicative items to conclude that subjects were categorising by the task-irrelevant dimension that was misaligned with the task. We call this performance criterion the non-conformant-categorisation criterion and subjects that reached it non-conformant learners for their behaviour does not conforms to what is expected from noisy learners that entertain the task-appropriate, but instead the task-inappropriate, representation. We applied a significance level of 0.05 to all statistical tests.

\subsubsection{Calibration studies}

Unsupervised calibration study The aim of the unsupervised calibration study was simply to confirm that subjects would attend to the salient, task-irrelevant dimension in the absence of feedback and that they would not recover the discrete group structure.

Subjects in the unsupervised condition were free to choose the key assignment for the two categories themselves. Thus, in order to combine the results across subjects for statistical analysis, we had to re-map these assignments into a common reference. Since, by definition, performance on unindicative items did not distinguish attention to task-relevant or task-irrelevant features, these provide the obvious reference. Therefore, response keys were re-mapped for each subject such that the mapping would maximise accuracy on these unindicative items.

Comparing subjects' accuracies against the performance criteria with this reference mapping, we found that $71 \%$ of subjects categorised according to the taskirrelevant dimension (by the $60 / 40 \%$ criterion described above). In fact, these subjects' approached the non-conformant-categorisation criterion quickly within the first two blocks. One-sided, one-sample permutation tests revealed that these subjects already performed above chance on indicative items $(p=0.006)$ and below chance on unindicative items $(p=0.001)$ on the very first block.

Only one subject passed the conformant-categorisation criterion. The remaining $21 \%$ of subjects did not meet either of the performance criteria and thus did not show behaviour consistent with a categorisation according to either salient or subtle stimulus features.

In summary, the results from this calibration study confirmed that the majority of subjects attended to the salient, task-irrelevant dimension and did not recover the discrete group structure if feedback was absent. 


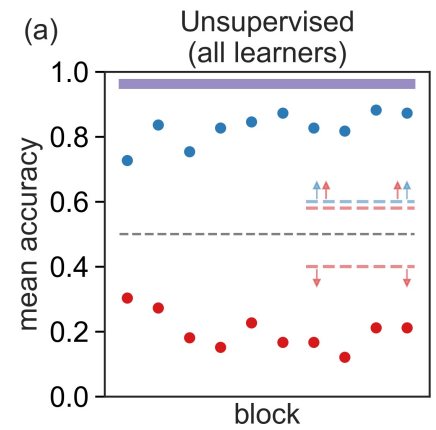

(b) $\begin{gathered}\text { Supervised } \\ \text { (fast learners) }\end{gathered}$

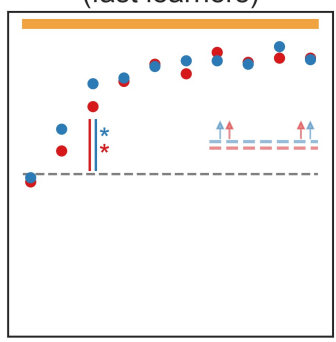

Semi-supervised

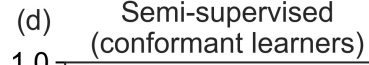

unindicative

- items

indicative

- items

unsupervised

block

supervised

block

categorisation

criterion

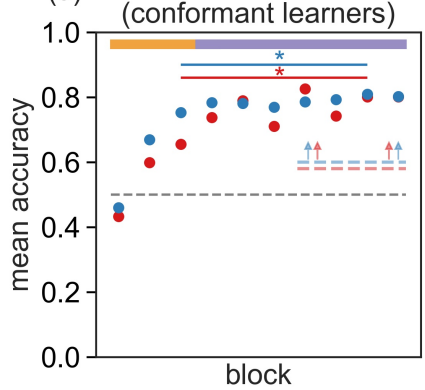

(c) Supervised (slow learners)

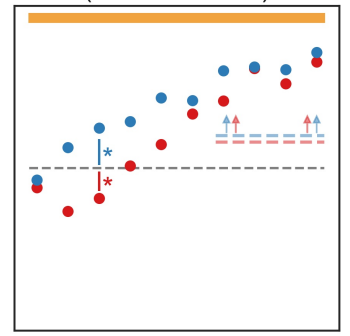

(e)

Semi-supervised

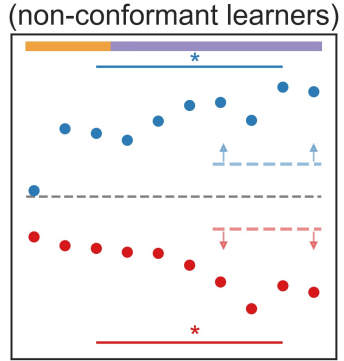

Figure 5: Average learning curves for subjects that passed the conformant and / or non-conformant performance criterion. The unsupervised and supervised calibration studies revealed a close qualitative match to predicted learning curves. (a) Unsupervised condition: Subjects tended to categorise by the task-irrelevant dimension. (b, c) Supervised condition: Half of the learners reached high performance on unindicative and indicative items by block 3 (fast learners) while the other half displayed a U-shaped learning curve on indicative items with below chance performance on block 3 (but above chance for unindicative items). (d, e) Semi-supervised condition: The benefit of unsupervised trials differed between groups of subjects. Subjects that met the conformant-categorisation criterion improved while non-conformant learners improved on unindicative but worsened on indicative items. The learning curves provide a close qualitative match to the predictions we made for fast and slow learners, but note that subjects here are split not by learning speed, but instead by the performance criterion applied to the last four blocks.

Supervised calibration study Having confirmed that subjects attended to the stimulus irrelevant dimension in the absence of feedback, the aim of the supervised calibration study was to assess whether feedback would help subjects to perform the task correctly and to assess the inter-individual variability in the speed of learning with feedback. In particular we sought to determine the time point in the experiment at which the subjects varied greatly in their performance on indicative items so that we could set the time of feedback withdrawal for the semi-supervised experiment 
accordingly.

We found that feedback indeed increased the proportion of subjects that would pass the conformant-categorisation criterion and that the variability between subjects was indeed large in the beginning of the experiment with some subjects even showing below chance performance as we expected for slow learning subjects. We established this with a small sample of subjects (see supplementary material), but report a replication with a larger sample (conducted after the semi-supervised experiment) here for the purpose of better illustration. With respect to the aim of this calibration study, the two samples have the same characteristics.

When trained with feedback throughout the experiment, $34 \%$ of subjects passed the conformant-categorisation criterion; $6 \%$ of the remaining subjects passed the non-conformant-categorisation criterion while the rest displayed behaviour inconsistent with either categorisation. It should be noted that this is a significantly lower percentage than the $74 \%$ of conformant learners observed in the smaller sample that we had collected at the earlier time point. The differences between samples likely reflect changes in the MTurk population following the effects of the corona pandemic (we will return to this in the discussion). However, the behaviour of those subjects who did learn to perform the task and passed our conformant-categorisation criterion was the same across both samples in all important respects, and we will thus here only consider the data from the 53 subjects that learnt to perform the task in the larger sample.

We found that the first block on which subjects who learned to perform the task reached $60 \%$ accuracy on both indicative and unindicative items was, on average, between blocks 3 and 4 (with the median being block 3). In fact, $53 \%$ of these subjects reached this performance level within the first three blocks (fast learners), while the other $47 \%$ (slow learners) required more training blocks. Performing onesided, one-sample permutation tests on subjects' accuracies on block 3 revealed that, in line with the desired learning curves, fast learners were already above chance level performance on indicative and unindicative items (both $p<0.001$ ). It can also be seen qualitatively that performance was lower on indicative compared to unindicative items as expected, although this effect was not statistically significant $(p=0.057)$.

For the slow learners, we also observed a close qualitative fit with their predicted (see Fig. 3) learning curve: subjects' performance on unindicative items monotonically increased while performance on indicative items was U-shaped. Importantly, one-sided, one-sample permutations tests confirmed that performance on unindicative items was above chance level on block $3(p=0.008)$, while it was below chance level for indicative items $(p=0.02)$.

In summary, the results from this calibration study confirmed that although most subjects initially attended to the task-irrelevant dimension, it was possible to learn the true category structure with feedback (noting that the percentage of people who could successfully solve the task differed between our two testing points as 
noted above). In addition, slowly learning subjects displayed a U-shaped learning curve (which, to our knowledge, is the first demonstration of such learning in a categorisation tasks with adults). In particular, subjects' learning curves revealed that performance varied greatly between subjects on the third training block making it a good candidate after which to withdraw feedback: learners who already attend to the task-relevant dimension, would be expected to improve even without feedback while slower learners would be expected to get worse as they still attend to the taskirrelevant dimension at this time.

\subsubsection{Semi-supervised Experiment}

The aim of the semi-supervised experiment was to test our semi-supervised representationto-task alignment hypothesis by assessing the impact that the representations subjects entertained (assessed by their momentary performance on (un-)indicative items) at the point of feedback withdrawal would have on subsequent unsupervised learning in this task. For the test to work, individual subjects would need to be differentially affected by the feedback withdrawal.

To assess any such differential effect on performance, we first grouped subjects as we did in the calibration studies. We divided subjects depending on whether they passed the conformant-categorisation criterion (27\%, conformant learners), the nonconformant-categorisation criterion (27\%, non-conformant learners), or did not show behaviour consistent with either categorisation (46\%, other subjects) at the end of the semi-supervised experiment.

We will report how conformant, non-conformant and other subjects were differentially impacted by unsupervised exposure and will refer to supplementary analyses that assess the degree to which performance change after feedback withdrawal could even be predicted from initial performance on supervised trials.

For the subjects who reached conformant or non-conformant end states of learning, we first observed a close qualitative fit between the average learning curves in both groups and our predictions for fast and slow learners. The group of conformant subjects defined by their good performance on indicative and unindicative items at the end of the experiment, already showed above chance performance on both item types before feedback withdrawal. Furthermore, performance seems to increase between the last supervised block and the end of the unsupervised training. In contrast, the group of non-conformant subjects who performed well on unindicative but poorly on indicative items at the end of the experiment, showed this trend already before feedback withdrawal. After that point, the performance difference on these items further increased. That is, conformant subjects displayed the performance characteristics we predicted for fast learners, while non-conformant subjects displayed the performance characteristics we predicted for slow learners (see bottom panels of Fig. 3 and 5 for comparison). The other subjects that did not reach either performance criterion displayed a flat curve with minimal deviations from chance across all blocks (see supplementary material). 
This qualitative observation was confirmed by statistical tests which revealed that, indeed, performance on the last supervised block (block 3) was above chance for unindicative items in both conformant and non-conformant groups, and that performance on indicative items was above chance for the conformant, but below chance for in the non-conformant, group $(p<0.001$ in all cases). The performance of the remaining subjects on block 3 was not significantly different form chance as confirmed by a two-sided, one sample permutation test ( $p=0.5$ for unindicative items, $p=0.79$ for indicative items).

We then tested specifically whether subjects' performance improved or degraded during the unsupervised phase. To evaluate this, we measured the direction of performance change (improvement, worsening or no change) from the last supervised block (block 3) to the last unsupervised block that, due to the alternating nature of consecutive blocks, contained the exact same item structure (block 9). We found that performance of the conformant and non-conformant learners diverged on the indicative items over the course of the experiment while performance on the unindicative items increased in both subject groups (Fig. 6). That is, a one-sample permutation test confirmed that the majority of conformant learners improved on unindicative items $(50 \%, p=0.02)$ as well as indicative items $(60 \%, p=0.002)$. While the majority of non-conformant learners also improved on unindicative items $(62 \%$, $p=0.006)$, the majority of them worsened on indicative items $(55 \%, p=0.01)$. It is also worth noting that performance did not change between these two blocks in about a quarter of subjects (conformant learners: $29 \%$ for unindicative and $21 \%$ for indicative items; non-conformant learners: $14 \%$ for unindicative and $24 \%$ for indicative items). Of the remaining subjects that did not pass either performance criterion $51 \%$ worsened, $40 \%$ improved and $8 \%$ did not change performance on unindicative items. Similarly, $38 \%$ worsened, $38 \%$ improved and $24 \%$ did not change performance on indicative items. As can be expected from the similar proportions of subjects that improved and worsened, two-sided one-sample permutation tests revealed that this subject group did not significantly change in performance over the unsupervised phase ( $p \geq 0.4$ for both item types). 


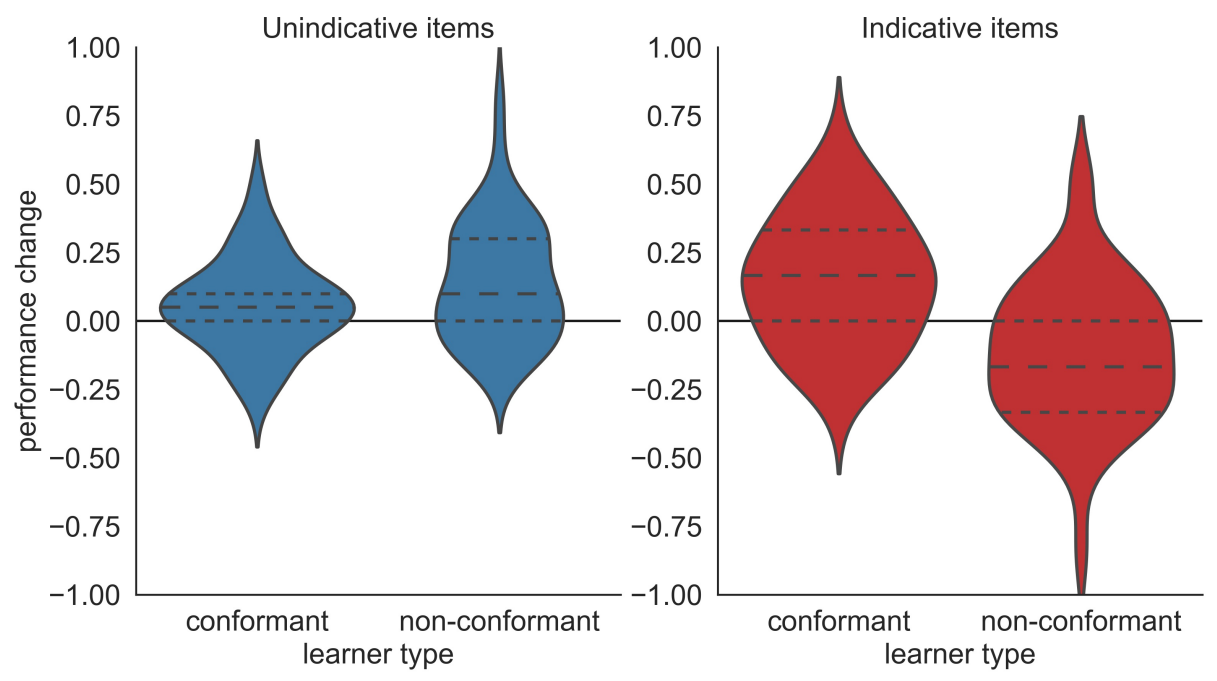

Figure 6: Percentiles of learners in the semi-supervised experiment that either improved (positive change), worsened (negative change) or maintained (no change) performance over the unsupervised phase (from block 3 to block 9). The majority of conformant and non-conformant learners improved on unindicative items. By contrast, the majority of conformant learners also improved on unindicative items whereas the majority of non-conformant learners worsened demonstrating that unsupervised trials can have opposing effects on task performance.

To summarise, these results demonstrate opposite effects of unsupervised learning expressed in the diverging performance levels on indiciative items that are indeed consistent with our predictions. Because results rely on a post-hoc split of subjects that depends on our arbitrarily set performance criterion (60/60 and 60/40 for conformant and non-conformant categorisation respectively) that defines the subject groups we compare here, we validated the robustness of these effects under two stricter performance criteria (see supplementary material for details).

These analyses confirmed that the significant effect of improvement and worsening on indicative items is robust to different learning criteria which supports the key experimental result demonstrating that performance of conformant and nonconformant learners diverges over the unsupervised phase. This is true despite ceiling effects that can be observed in the data (i.e. subjects who performed near perfectly already at the end of the supervised phase were more likely to decrease in performance; see supplementary material). Improvement on unindicative items in both groups (which we expected to find but was not key to revealing opposing effect of unsupervised exposure) is significant under two out of three learning criteria for both groups and thus slightly less robust. Inspecting the data, the smaller effect in the unindicative items is likely due to stronger ceiling effects. That is, subjects 
had high average performance on unindicative items on block 3, leaving little room for improvement during the unsupervised phase and thus confirming the point that they are less useful than the indicative items to evaluate differential effects in our experimental design.

In addition, we performed supplementary analyses to assess whether performance in the initial supervised phase was even predictive of later performance as should be the case if performance is not too noisy (see supplementary material). In summary, the results are consistent with our original predictions. Subjects with high initial accuracy were more likely to improve while those with low initial accuracy were more likely to worsen. However, predictive accuracy of performance change was limited due to noisy performance and different behavioural patterns across subjects.

To summarise, we found robust evidence that subjects who reached different end states of learning (aligned or not aligned with the task) were differentially impacted by unsupervised exposure. The degree to which performance change was predictable from initial performance was limited due to behavioural noise. 


\section{General Discussion}

We sought to test our hypothesis that unsupervised training can either help or hurt learning in a particular task depending on the degree to which internal representations of the inputs are aligned with this task. We evaluated this by investigating the effect of feedback withdrawal on subjects that solved the same categorisation problem but differed in their internal representations at the time of withdrawal due to difference in learning speed. We presented data consistent with the prediction that unsupervised learning can have opposite effects on human performance even within the same task.

In line with our hypothesis - that the direction of this effect would depend on the performance of individual subjects, and hence their internal representations, at the point of feedback withdrawal - we found a close qualitative match between our predictions and group-level performance. On average, the subjects who benefited from unsupervised trials were those who performed well at the time of feedback withdrawal, putatively refining and strengthening their broadly correct categorisations. Conversely, subjects whose performance degraded following unsupervised trials were those who performed poorly at the time of feedback withdrawal, putatively by selfreinforcing their incorrect categorisation. That we could evaluate this by assessing subjects' performance on those items that were indicative of the representation they entertained was a key feature of our study design. Crucially, while performance on these items increased for the first group, performance on them of the second group deteriorated. While we were also able to predict individual subjects' future performance from their initial performance during the supervised training in some respects, this was only possible to a moderate degree.

While on the whole, research on semi-supervised categorisation has reported mixed effects across experiments, it had not previously been shown that unsupervised learning can have opposite effects even within the exact same category learning task. However, experiments in related areas of human research have also observed that unsupervised trials can have seemingly opposing effects within almost identical tasks. In perceptual learning, subjects seem to improve on easy discriminations without feedback, but not if tasks are difficult [Liu et al., 2010, Dosher and Lu, 2017]. Moreover, in studies investigating U-shaped language learning of irregular English plurals, it has been shown that younger, error-prone, children will perform worse after unsupervised exposure to plurals while older, more proficient, children will improve given the same unsupervised training [Ramscar and Yarlett, 2007, Ramscar et al., 2013]. In addition, pre-exposure experiments in animals and humans have shown that initial, unsupervised exposure can either facilitate or retard later supervised learning depending on the exact stimulus material used [Saksida, 1999, Wills et al., 2004]. While these tasks were not designed to address the question of whether subjects can utilise unsupervised distributional information, and hence answer different questions to the one central to many semi-supervised categorisa- 
tion experiments, the general learning problem however appears similar. Thus, to summarise, while opposite effects of unsupervised learning on category learning may seem surprising at first, our results resonate with a larger body of work that has reported similar phenomena in different learning contexts.

\subsection{Internal Representations}

Apart from the empirical results in other literatures, opposite effects of unsupervised learning may also be expected from the same theoretical perspective that gave rise to the predictions that motivated the first semi-supervised categorisation experiments [Zhu et al., 2007]. It is well-known that unsupervised data can only boost performance of semi-supervised algorithms beyond supervised data under certain assumptions [Chapelle et al., 2006]. One popular instance in machine learning is the cluster assumption: that the data form discrete groups (i.e. clusters), data in the same group belong to the same category and equivalently that category boundaries lie in low density regions. While rarely discussed explicitly, the majority of behavioural semi-supervised categorisation tasks were designed in accordance with this intuitive prerequisite for semi-supervised learning to be successful. In these studies, unsupervised and supervised stimuli are typically drawn from such wellbehaved category distributions to test the prediction that subjects' would benefit from the unsupervised trials in much the same way that a semi-supervised algorithm would be expected to improve given that the theoretical assumptions appeared to have been met. However, as pointed out, the mixed results in these studies are at odds with this simple prediction.

Although it appears that the cluster assumption in these studies was met, and hence that subjects' should be able to benefit from semi-supervised learning in theory, our work shows that a subtle, but important, difference exists when studying human learning. For semi-supervised learning to benefit human performance in an experimental task, even theoretically, it is not sufficient for stimuli to separate into groups in the experimenter-defined space. Instead, crucially, subjects' internal representation of the stimuli, which will typically be compressed, needs to be sufficiently aligned with the geometry of the experimenter-defined task space in order for subjects to harness statistical information in a task-appropriate manner at all. While predictions and interpretations of the vast majority of previous studies on semi-supervised categorisation have implicitly assumed a one-to-one mapping between the experimenter-defined stimulus space and subjects' internal representational space, our results contribute evidence that this is not only generally untrue but that experimenter-defined dimensions may indeed be insufficient to predict performance.

Concretely, that a one-to-one mapping cannot be taken for granted was demonstrated by our subjects' inability to recover the group structure in the absence of feedback. Instead, they readily divided stimuli into categories along a continuously varying dimension such that their category boundary passed through a high-density 
region in experimenter-defined space. Furthermore, subjects whose supervised learning was slow exhibited U-shaped learning curves, highlighting the fact that representations of the stimulus material can change within tasks and at different rates across subjects. We hypothesised that, because of these different rates of representational change, only subjects who had learned to attend to the subtle, task-relevant dimension would be able to improve when feedback was withdrawn because the distributional information about this discrete group structure was available only to them. By contrast, it would be unavailable to subjects attending to the salient but task-irrelevant dimension. This is consistent with the prediction made by Rogers et al. [2010] and Gibson et al. [2013] that selective attention will change which distributional information is available to the learner as well as the prediction made by Hammer [2015] that more feedback is needed if stimulus clusters differ along subtle rather than salient dimensions.

If predictions about subjects' learning indeed hinge on their internal representations - shaped by prior experience and learning in the task itself - in relation to the experimenter-defined space that defines success in the task, then one reason why predictions do not generalise across semi-supervised categorisation experiments may simply be that this aspect was not assessed and controlled for in the different tasks employed [see also Rogers et al., 2010]. It is also worth noting that the problem of mismatch between experimenter-defined and internal representations is not unique to semi-supervised learning, but our concern joins recent work from research on category learning, perceptual learning and visual memory arguing that the assumption of a one-to-one mapping between external and internal representations of the stimulus material severely limits the predictions made by, and conclusion drawn from, wellestablished theories [Roark et al., 2020, Zaman et al., 2020, Schurgin et al., 2020]. As for these studies, we also suggest that assessing and quantifying representations may help better understand and predict behaviour in semi-supervised tasks. Given the increasing interest in providing methods for assessing internal representations, future work will be able to draw on recently developed methods such as techniques for constructing representational spaces from subjects' behavioural judgements [e.g. Houlsby et al., 2013, Nosofsky et al., 2018, Roads and Mozer, submitted, Hebart et al., 2020] or using deep neural networks to approximate human representations directly [Battleday et al., 2019, Ma and Peters, 2020].

\subsection{Mechanism}

While we hypothesised that good performance should lead to improvements, and showed results consistent with this prediction, the question remains what mechanisms could underlie such learning and thus how the learning process could be formalised computationally.

Several models employed to account for the category boundary shifts observed in some of the semi-supervised categorisation experiments, relied on mechanisms of self-training [Gibson et al., 2013]. Self-training algorithms learn from their own 
predictions (or responses) in lieu of external labels if these are unavailable [Zhu and Goldberg, 2009]. Given that the learning problems and tasks are fairly similar to the one reported here, it appears likely that such a mechanism may also underlie the learning of our subjects. In particular, because a self-training mechanism naturally predicts that improvement is only possible if the task is already appropriately represented (because in that case subjects' learn from their mostly correct predictions), and detrimental otherwise (because internal predictions are mainly incorrect) [see also Oymak and Gulcu, 2020, for theoretical insights].

While self-training algorithms appear to be promising model candidates because they can capture the general phenomena described here, it would also be possible that subjects make some more elaborate estimation of the density of their inputs (see e.g. the Dirichlet Process Mixture Model by Zhu et al. [2010]), albeit with respect to the representation they currently entertain.

Independent of the underlying mechanism, modelling of individual subjects' trial-by-trial representational change and learning of the category labels will not be without challenges especially when behaviour is noisy and stimuli are complex.

\subsection{Limitations and Future Directions}

While developing and testing hypotheses that account for internal representations appears to be an important direction, assessing these representations is tricky, and so this will likely be challenging. This challenge applies to modelling representational learning itself, and also other potential directions for extending the present work.

In the remaining discussion we will focus on how predictability and generalisability can be complicated by the need to assess representations, how this expressed in our study and how this can be addressed in the future.

\subsubsection{Predictability}

That testing predictions when representations need to be assessed is challenging, was also reflected in the work presented here. For example, we assumed that subjects' access to appropriate representations would be expressed as above-chance performance which would, in turn, provide the basis for performance improvements also in the absence of feedback. If performance had been a noise-free indicator of internal representations, it should have been possible to predict subjects' future performance from their momentary performance above and beyond the group-level differences we found. While we were able to predict whether subjects would perform well or poorly on the task after feedback withdrawal, more specific predictions about improvement or worsening during the unsupervised phase based on momentary performance were complicated by ceiling effects as well as regression to the mean performance.

Anticipating some of these challenges, we sought to design the experiment to ensure that individual subjects would undergo slow and gradual learning, with accuracies being reflective of underlying representations. In order to obtain the opposite 
directions of performance change between the different subject groups as we did, learning had to be sufficiently slow before reaching an asymptote because rapid, step-like learning curves reported in other experiments [e.g. Gallistel et al., 2004, Smith and Ell, 2015] would not have allowed us to make inferences about continued learning. This created a trade-off between the shape of learning curves and learnability, which likely resulted in the many subjects not learning the intended categorisations despite the stimulus material and task being rather simplistic. At the same time, other subjects approached perfection quickly leading to ceiling effects. These observations demonstrate that, even in such simple experimental tasks like the one employed here, inter-individual variability is large and subjects' momentary accuracy is only a noisy correlate of their underlying internal representations with both sources of noise magnified when testing online.

In summary, while our study was built on the fact that every subject is unique (representing inputs differently and changing representations at different rates), this fact also complicated the study. One future avenue to increase predictability of results and generalisability to a larger population of subjects, would be to design experiments that tailor task difficulty to individual subjects (e.g. by using staircase procedures). This could help increase the number of subjects that are engaged in the task, can pass the learning criterion and achieve a gradual learning curve while reducing the speed of learning therefore also preventing ceiling effects. That individually adapted task difficulty may be necessary especially when testing online appears particularly likely given that we observed fluctuations in the percentage of subjects that were able to reach our learning criterion in supervised calibration study at different times during the corona pandemic. This informally suggests that optimal experimental parameters for this task may be population dependent.

\subsubsection{Generalisability}

We started out by proposing that the alignment between internal representations and tasks is important for predicting the effect of unsupervised information. Because our task required subjects mainly to suppress the salient, task-irrelevant dimension a simple form of representational change - and to learn the systematic mapping from the appendages to the category labels, the idea of alignment between representation and task is simple in this case. While learning to distinguish sheep from goats may be equally simple once the correct feature is known, many natural learning tasks that humans face require more extensive representational learning in more sophisticated task structures. To make any precise and general statements about what it means for internal representations to be sufficiently aligned to the task, it will not only be necessary to capture internal representations accurately, but also the learning mechanisms which define the way in which representations change over the course of learning (including unsupervised representational learning; Hinton and Sejnowski [1999]). That is, to test the general predictions our hypothesis makes in more complicated task, statements about alignment and their formalisation will 
need to take into account this additional complexity.

In addition to this, it is worth keeping in mind that there may well be additional factors impacting semi-supervised learning in humans that go beyond computational factors like representation and distributional information, such as for example the learner's motivation. This is particularly true when considering learning tasks outside the lab in which behaviour is far less constrained. For instance, a successful tutoring application not only needs to optimise presentation of content to maximise performance but also needs to maintain the learner's motivation so they continue to practice in the future. Since much of human learning, even in an educational context, often takes place without external supervision, it is important to better understand the complex interplay of all the relevant factors and how they contribute to semi-supervised learning for insights into this have the potential to help design and improve tutoring systems and instruction.

\section{Conclusions}

Humans learn both with and without explicit feedback, akin to the semi-supervised learning of machines. However, experimental results investigating the benefit of unsupervised learning for human performance have been mixed. Here, we tested the hypothesis that one critical factor determining the effect of unsupervised information on learning is the alignment between internal representations and task-relevant feature dimensions. In line with this, we showed that unsupervised training can lead to mixed effects across subjects even within the exact same task due to individual differences in representations and learning. Our results imply that understanding how humans learn under different feedback conditions requires assessment of the representations that subjects entertain for the materials involved in these tasks. These considerations apply to both studies in the lab and the design of tutoring systems and instruction. 


\section{Data and Code}

The data, experimental and analysis code will be made available upon publication.

\section{Acknowledgements}

We thank Brett Roads for helpful discussions on running online experiments and Noémi Éltető for reviewing the code associated with the analyses in this paper. This work was supported by the Gatsby Charitable Foundation (FB); the Max Planck Society (FB, PD); the Alexander von Humboldt Foundation (PD); and the NIH Grant 1P01HD080679, Wellcome Trust Investigator Award WT106931MA, and Royal Society Wolfson Fellowship 183029 (BCL). 


\section{References}

Ruairidh M Battleday, Joshua C Peterson, and Thomas L Griffiths. Capturing human categorization of natural images at scale by combining deep networks and cognitive models. arXiv preprint arXiv:1904.12690, 2019.

O. Chapelle, B. Schölkopf, and A. Zien. Semi-supervised Learning. Adaptive computation and machine learning. MIT Press, 2006. ISBN 9780262033589. URL https://books.google.de/books?id=kfquQgAACAAJ.

Barbara Dosher and Zhong-Lin Lu. Visual perceptual learning and models. Annual Review of Vision Science, 3:343-363, 2017.

Charles R Gallistel, Stephen Fairhurst, and Peter Balsam. The learning curve: implications of a quantitative analysis. Proceedings of the National Academy of Sciences, 101(36):13124-13131, 2004.

Bryan R Gibson, Timothy T Rogers, and Xiaojin Zhu. Human semi-supervised learning. Topics in cognitive science, 5(1):132-172, 2013.

Bryan R Gibson, Timothy T Rogers, Chuck Kalish, and Xiaojin Zhu. What causes category-shifting in human semi-supervised learning? In CogSci, 2015.

Rubi Hammer. Impact of feature saliency on visual category learning. Frontiers in psychology, 6:451, 2015.

Martin N Hebart, Charles Y Zheng, Francisco Pereira, and Chris I Baker. Revealing the multidimensional mental representations of natural objects underlying human similarity judgements. Nature Human Behaviour, 4(11):1173-1185, 2020.

Geoffrey E Hinton and Terrence Joseph Sejnowski, editors. Unsupervised learning: foundations of neural computation, 1999. MIT press.

Neil MT Houlsby, Ferenc Huszár, Mohammad M Ghassemi, Gergő Orbán, Daniel M Wolpert, and Máté Lengyel. Cognitive tomography reveals complex, taskindependent mental representations. Current Biology, 23(21):2169-2175, 2013.

Charles W Kalish, Sunae Kim, and Andrew G Young. How young children learn from examples: Descriptive and inferential problems. Cognitive science, 36(8): 1427-1448, 2012.

Charles W Kalish, XiaoJin Zhu, and Timothy T Rogers. Drift in children's categories: when experienced distributions conflict with prior learning. Developmental science, 18(6):940-956, 2015.

Brenden Lake and James McClelland. Estimating the strength of unlabeled information during semi-supervised learning. In Proceedings of the Annual Meeting of the Cognitive Science Society, volume 33, 2011. 
Jiajuan Liu, Zhong-Lin Lu, and Barbara A Dosher. Augmented hebbian reweighting: Interactions between feedback and training accuracy in perceptual learning. Journal of vision, 10(10):29-29, 2010.

Wei Ji Ma and Benjamin Peters. A neural network walks into a lab: towards using deep nets as models for human behavior. arXiv preprint arXiv:2005.02181, 2020.

John V McDonnell, Carol A Jew, and Todd M Gureckis. Sparse category labels obstruct generalization of category membership. In Proceedings of the Annual Meeting of the Cognitive Science Society, volume 34, 2012.

Robert M Nosofsky, Craig A Sanders, Brian J Meagher, and Bruce J Douglas. Toward the development of a feature-space representation for a complex natural category domain. Behavior research methods, 50(2):530-556, 2018.

Samet Oymak and Talha Cihad Gulcu. Statistical and algorithmic insights for semisupervised learning with self-training. arXiv preprint arXiv:2006.11006, 2020.

John D. Patterson and Kenneth J. Kurtz. Semi-supervised learning: A role for similarity in generalization-based learning of relational categories. In Proceedings of the 40th Annual Conference of the Cognitive Science Society, 2018.

Kyle Peyton, Gregory A Huber, and Alexander Coppock. The generalizability of online experiments conducted during the covid-19 pandemic. 2020.

Michael Ramscar and Daniel Yarlett. Linguistic self-correction in the absence of feedback: A new approach to the logical problem of language acquisition. Cognitive science, 31(6):927-960, 2007.

Michael Ramscar, Daniel Yarlett, Melody Dye, Katie Denny, and Kirsten Thorpe. The effects of feature-label-order and their implications for symbolic learning. Cognitive science, 34(6):909-957, 2010.

Michael Ramscar, Melody Dye, and Stewart M McCauley. Error and expectation in language learning: The curious absence of" mouses" in adult speech. Language, pages 760-793, 2013.

Brett D Roads and Michael C Mozer. Obtaining psychological embeddings through joint kernel and metric learning. submitted.

Casey L Roark, David C Plaut, and Lori L Holt. A neural network model of the effect of prior experience with regularities on subsequent category learning. 2020.

Timothy Rogers, Charles Kalish, Bryan Gibson, Joseph Harrison, and Xiaojin Zhu. Semi-supervised learning is observed in a speeded but not an unspeeded 2 d categorization task. In Proceedings of the Annual Meeting of the Cognitive Science Society, volume 32, 2010. 
Lisa M Saksida. Effects of similarity and experience on discrimination learning: a nonassociative connectionist model of perceptual learning. Journal of Experimental Psychology: Animal Behavior Processes, 25(3):308, 1999.

Mark W Schurgin, John T Wixted, and Timothy F Brady. Psychophysical scaling reveals a unified theory of visual memory strength. Nature Human Behaviour, pages $1-17,2020$.

J David Smith and Shawn W Ell. One giant leap for categorizers: One small step for categorization theory. PloS one, 10(9):e0137334, 2015.

Katleen Vandist, Maarten De Schryver, and Yves Rosseel. Semisupervised category learning: The impact of feedback in learning the information-integration task. Attention, Perception, \& Psychophysics, 71(2):328-341, 2009.

Katleen Vandist, Gert Storms, and Eva Van den Bussche. Semisupervised category learning facilitates the development of automaticity. Attention, Perception, \& Psychophysics, 81(1):137-157, 2019.

Pepper Williams. Representational organization of multiple exemplars of object categories. Retrieved August, 23:2017, 1998.

Andy J Wills, Mark Suret, and Ian PL McLaren. The role of category structure in determining the effects of stimulus preexposure on categorization accuracy, 2004.

Jonas Zaman, Anastasia Chalkia, Ann-Kathrin Zenses, Antoine Selim Bilgin, Tom Beckers, Bram Vervliet, and Yannick Boddez. Perceptual variability: Implications for learning and generalization. Psychonomic Bulletin \&5 Review, August 2020. ISSN 1069-9384, 1531-5320. doi: 10.3758/s13423-020-01780-1. URL http:// link. springer.com/10.3758/s13423-020-01780-1.

Xiaojin Zhu and Andrew B Goldberg. Introduction to semi-supervised learning. Synthesis lectures on artificial intelligence and machine learning, 3(1):1-130, 2009.

Xiaojin Zhu, Timothy Rogers, Ruichen Qian, and Chuck Kalish. Humans perform semi-supervised classification too. In Twenty-Second AAAI Conference on Artificial Intelligence (AAAI-07), 2007.

Xiaojin Zhu, Bryan R Gibson, Kwang-Sung Jun, Timothy T Rogers, Joseph Harrison, and Chuck Kalish. Cognitive models of test-item effects in human category learning. In Proceedings of the 27th International Conference on Machine Learning (ICML), pages 1247-1254, 2010. 


\section{Supplementary Information}

\subsection{Data sample comparison for supervised calibration study}

An initial sample for the supervised calibration study was collected in early March 2020. As described in the main text, this served two functions. Firstly, it served to validate that feedback helped subjects to attend to the subtle, task-relevant stimulus features so as to learn to perform the task correctly. Secondly, it served to determine the optimal time to withdraw feedback in the semi-supervised experiment.

The collected data showed that the majority of subjects $(74 \%)$ were able to reach our performance criterion by the end of the experiment which was evidence for supervision to improve learning in this task. It also showed that subjects reached $60 \%$ accuracy on indicative and unindicative item types on average between block 3 and 4 (with the median on block 3 ) and that accuracy on indicative items varied greatly on block 3. Performing one-sided, one-sample permutation tests on subjects' accuracies on block 3 revealed that, in line with the desired learning curves, fast learners were already above chance level performance on indicative and unindicative items (both $p<0.001$ ). For the slow learners, we also observed a close qualitative fit with their predicted learning curve: subjects' performance on unindicative items increased while performance on indicative items appeared to be below chance early in learning. One-sided, one-sample permutations tests confirmed that performance on unindicative items was above chance level on block $3(p=0.03)$, however performance on indicative items was not significantly below chance level $(p=0.16)$.

Since the learning curves supported the main function this calibration study served to provide (establishing that feedback aided learning and observing fast and slow learning), this sample motivated the choice of withdrawing feedback on block 3 in the semi-supervised experiment (collected between late March and early May 2020). However, because the sample collected was small (34 subjects after exclusion), we aimed to replicate the learning curves with a larger sample for illustrational purposes. Thus, we collected a larger, independent, sample between late July and late August (157 subjects after exclusion was applied, as discussed in the main text).

To our surprise, the two samples differed significantly in the percentage of subjects who passed the learning criterion. A two-sided, two sample permutation test showed that the percentage of learners in the later sample was significantly lower $(p<0.001)$. However, we were primarily interested in the learning curves of those subjects that did learn to perform the task well. In order to report those learning curves, we sought to ensure that the samples did not differ with respect to their characteristics.

First, we ensured that learners in both samples needed the same time to reach $60 \%$ accuracy on both item types on average. Two-sided, two-sample permutation tests confirmed that subjects did not differ in the time they needed to achieve this performance $(p=0.14)$ and also that the percentage of fast / slow learners did not differ significantly $(p=0.41)$. Two-sided, two sample permutation tests also 
confirmed that the accuracy achieved on the last four blocks did not differ for fast and slow learners on either item type (all $p>0.13$ ).

Presumably, the difference in percentage of learners is due to changes in the subject population due to the Corona pandemic with fewer experienced or engaged subjects than before [Peyton et al., 2020]. Additional evidence for changes in how engaged subjects were in the task was further provided by a significantly higher percentage of subjects that received one or two performance warnings in the later sample ( $p=0.045$ two-sided, two-sample permutation test).

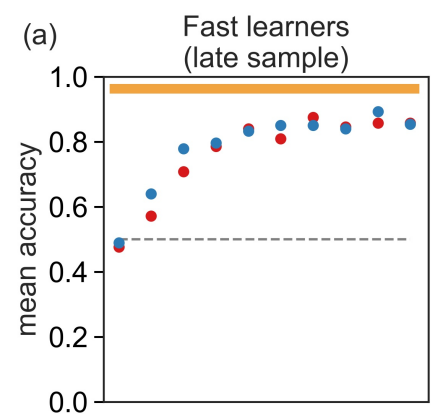

(b) Slow learners
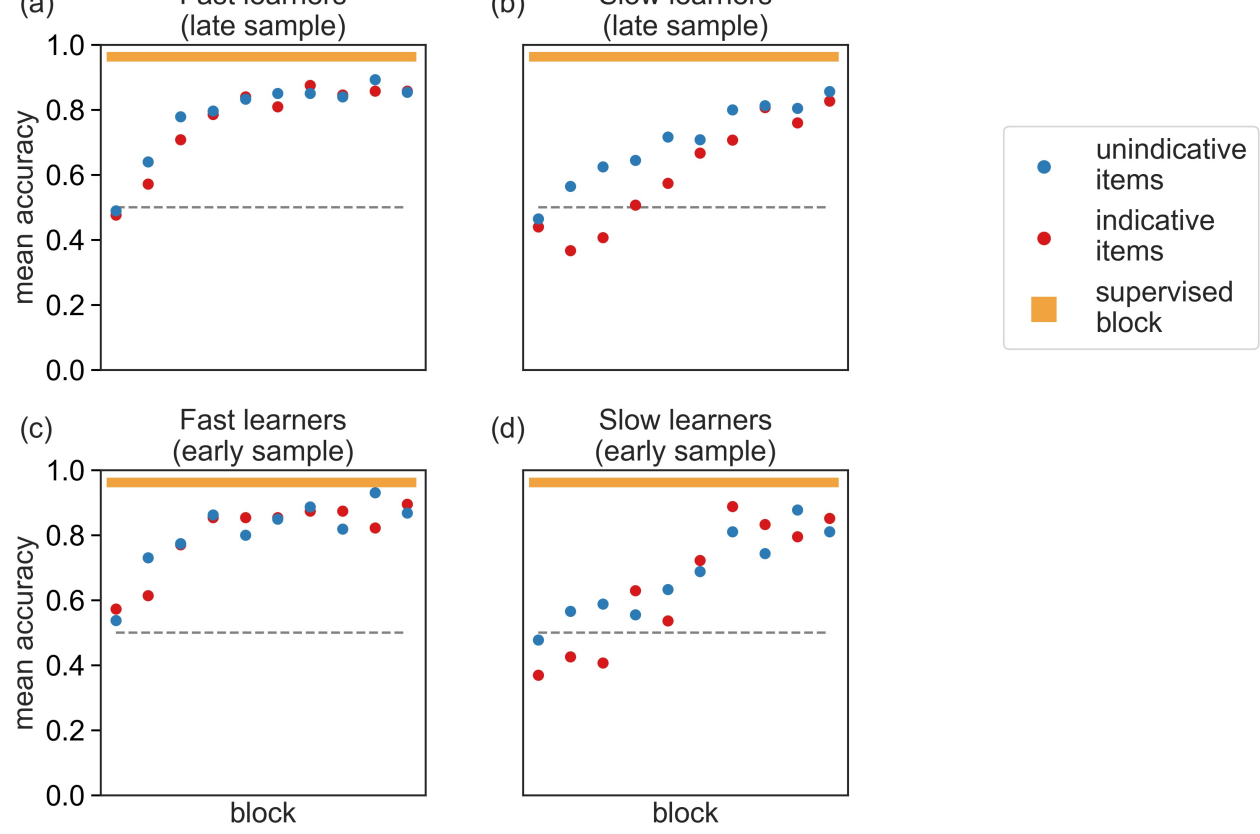

(d) Slow learners

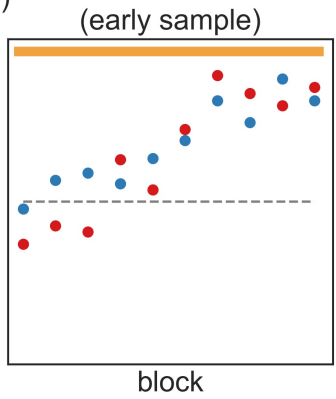

Figure 7: Average performance of subjects trained with full feedback in the early and late calibration sample. The late sample is reported in the main text.

\begin{tabular}{lccc}
\hline & Subjects & Learners (\%) & Subjects w/o performance warning (\%) \\
\hline Early sample & 34 & $25(74 \%)$ & $28(82 \%)$ \\
Late sample & 157 & $53(34 \%)$ & $101(64 \%)$ \\
\hline
\end{tabular}

Table 1: Number of subjects that were tested with full supervision in the early and late calibration sample, that reached the correct-categorisation criterion, and that completed the task without receiving any performance warning. 


\subsection{Learning curves of subjects not reaching learning criteria}

We display the learning curves of the conformant and non-conformant learners in the semi-supervised experiment in the main text. We also report that the other subjects which did no reach either performance criterion showed no significant improvement or worsening over the course of the unsupervised phase. This can also be seen qualitatively (Fig. 8).

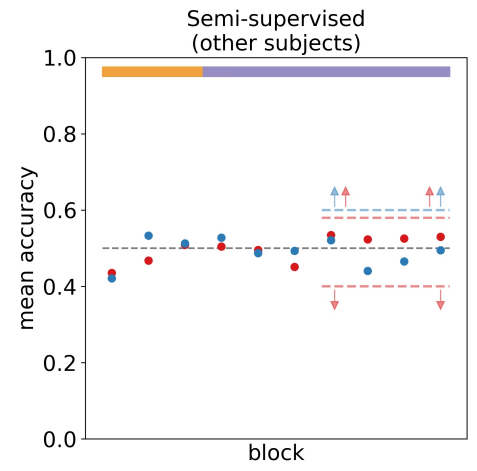

unindicative

items

indicative

- items

unsupervised

block

supervised

block

-- categorisation
--- criterion

Figure 8: Average performance of subjects in the semi-supervised experiment that did not reach either the conformant nor the non-conformant performance criterion. 


\begin{tabular}{|c|c|c|c|c|c|c|}
\hline \multirow[t]{3}{*}{ Criterion } & \multicolumn{3}{|c|}{ Conformant Learners } & \multicolumn{3}{|c|}{ Non-conformant Learners } \\
\hline & \multirow[t]{2}{*}{ \# subjects } & \multicolumn{2}{|c|}{ p-Value } & \multirow{2}{*}{ \# subjects } & \multicolumn{2}{|c|}{ p-Value } \\
\hline & & indicative & unindicative & & indicative & unindicative \\
\hline $60 / 60 \& 60 / 40$ & 42 & 0.002 & 0.02 & 42 & 0.01 & 0.006 \\
\hline $70 / 70 \& 70 / 30$ & 30 & 0.02 & 0.13 & 29 & 0.02 & 0.004 \\
\hline $80 / 80 \& 80 / 20$ & 15 & 0.047 & 0.04 & 14 & 0.03 & 0.09 \\
\hline
\end{tabular}

Table 2: P-values indicating whether a significant proportion of subjects changed performance from block 3 to block 9 under different learning criteria in accordance with the predicted direction of performance change. P-values indicate whether conformant learners improved on indicative and unindicative items and whether nonconformant learners improved on unindicative and worsened on indicative items.

\subsection{Robustness of semi-supervised effects depending on learning criterion}

In order to assess whether the unsupervised learning effects observed between conformant and non-conformant learners in the semi-supervised experiment were due to the arbitrary learning criterion chosen, we performed the same analysis with two stricter performance criteria (table 2). Again, we tested whether a significant proportion of conformant learners improved on unidicative and indicative items, and whether a significant proportion of non-conformant learners improved on unindicative but worsened on inidcative items (all compared between block 3 and 9).

The results show that effects on indicative items are significant under all three learning criteria. Improvement on non-indicative items was significant only under two of the three learning criteria both for conformant and non-conformant learners (table 2). This is likely due to the fact that subjects improved on unindicative items faster and so where closer to ceiling at the end of the supervised phase leaving little room for improvement over the unsupervised phase. That is, the effect can be expected to be smaller than for indicative items. 


\subsection{Ceiling and regression to chance effects}

Data inspection revealed ceiling effects for conformant and non-conformant learners in the semi-supervised experiment. For unindicative items, subjects that had above $80 \%$ accuracy on block 3 , were likely to worsen in performance towards block 9 . For indicative items, we see that conformant learners were more likely to improve while non-conformant learners were more likely to worsen, but again with a reversal in this effect if initial performance was low $(\leq 0.17)$ or high $(\geq 0.83)$. However, it can also be seen that if initial performance was just above or below chance $(0.33$ and $0.67)$ the trend of improvement or worsening is very clear: if below chance, subjects were more likely to get worse, if above chance, subjects were more likely to improve (Fig. 9).
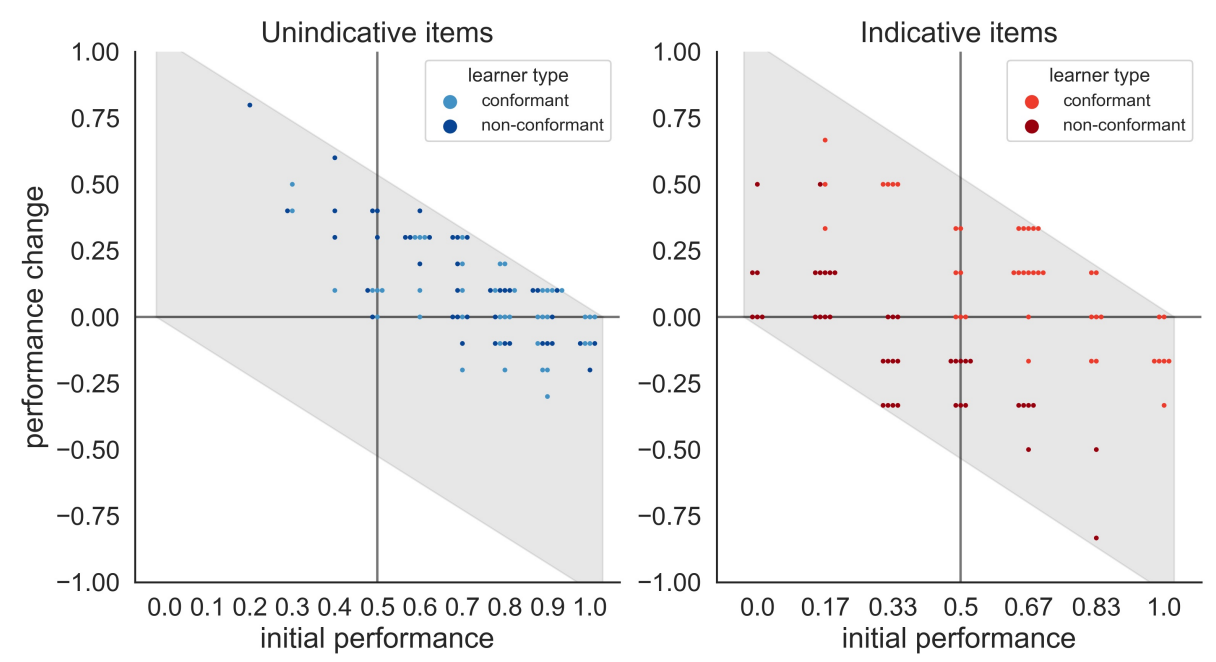

Figure 9: Initial performance on block 3 is shown against the performance change in comparison with block 9 for conformant and non-conformant learners in the semi-supervised experiment. Ceiling effects: subjects with low accuracy $(p \leq 0.17)$ on block 3 are more likely to improve slightly, while subjects with high accuracy $(p \geq 0.83)$ are more likely to worsen slightly.

On the other hand, the other subjects who did not reach either categorisation criterion by the end of the experiment showed a very different picture of results. For both indicative and unindicative items, the probability of improvement or worsening reverses at an initial performance of 0.5 indicating regression to chance performance. That is, almost no subjects with higher than chance performance on block 3 improved towards block 9 , and almost no subjects with lower than chance performance on block 3 worsened towards block 9 (Fig. 10). 

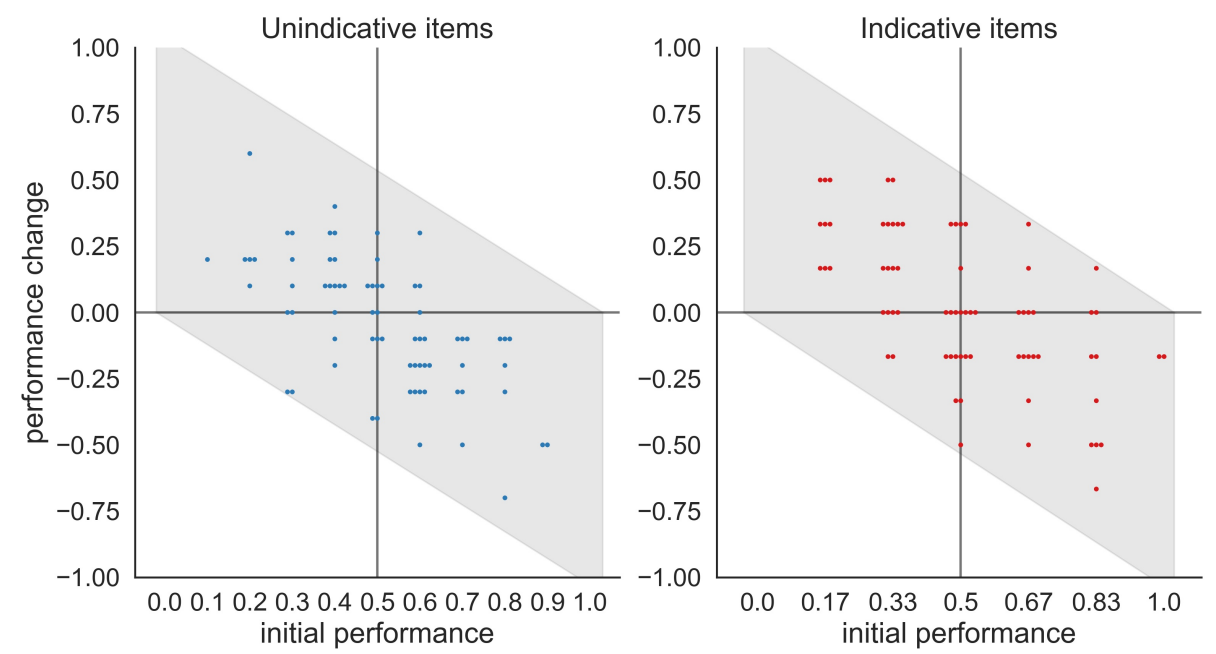

Figure 10: Initial performance on block 3 is shown against the performance change in comparison with block 9 for subjects that neither passed the conformant nor nonconformant performance criterion in the semi-supervised experiment. Regression to chance effects: subjects with below chance accuracy on block 3 were more likely to improve slightly, while subjects with above chance accuracy were more likely to worsen slightly.

\subsection{Predicting performance change from momentary performance}

We showed that opposite effects of unsupervised learning arose across the different subject groups. Since this relies on a post-hoc split of subjects into conformant, non-conformant and other subjects, we sought to assess whether performance in the initial supervised phase was even predictive of later performance as should be the case if performance is not too noisy.

We will begin with reporting results only for the conformant and non-conformant learners showing that our predictions are supported. We will then show that our predictions are also consistent with the data collected from the whole population of subjects (not including the subjects that did not pass the general exclusion criteria specified above). Reported logistic regression analyses include a constant bias term in addition to the predictors reported in the text.

\subsubsection{Conformant and non-conformant learners}

We begin to analyse how predictive momentary performance in the supervised phase was of future performance by restricting ourselves to conformant and non-confrmant learners first before analysing all subjects jointly.

Before turning to predicting performance change, we first sought to establish 
whether initial performance was at all predictive of the end-state of learning given the ceiling effects and fairly rapid learning observed that makes it difficult to distinguish performance change from noise. To test this we first performed a leaveone-out-cross-validation analysis (to avoid over-fitting) in which we employed a logistic regression model to predict whether subjects would pass the conformantcategorisation or non-conformant-categorisation criterion from their performance on indicative and unindicative items on block 3 . Predictions yielded $80 \%$ accuracy which was confirmed to be significantly above chance by a test permuting predicted group labels $(p<0.001)$.

Next, we sought to test specifically whether we could predict performance change from initial accuracy; that is, whether subjects would improve or worsen over the unsupervised phase as would be predicted if accuracy is a accurate correlate of the representations subjects entertained in this task. To minimise the impact of ceiling effects and other behavioural fluctuations onto predictions about performance change, we sought to predict whether individual subjects reached perfect or zero accuracy at least on one unsupervised block from subjects' initial performance on the last supervised block (block 3). We employed this stringent proxy for overall performance change since perfect performance by definition implies performance improvement or continuation in comparison to initial performance and zero accuracy implies worsening or continuation. It also served to ensure that the observed improvement and worsening reported for the two subject groups in the main text was not simply reflecting the post-hoc grouping by which the behaviour of atypical subjects (e.g. initially performing well, but performing poorly at the end of the experiment) could potentially dominate the results.

We first assessed the probability of reaching perfection for both item types separately. In total, $54 \%$ of subjects reached perfect accuracy on unindicative, and $37 \%$ on indicative items at least once during the unsupervised phase. To predict whether a subject would reach perfection, we fitted a logistic regression model for each item type capturing the probability of a subject reaching perfection given initial accuracy. The model revealed that the probability of perfect performance indeed significantly increased with initial performance $\left(\beta_{a c c}=3.8\right.$ with $p=0.003$ for unindicative items and $\beta_{a c c}=4.7$ with $p<0.001$ for indicative items). Applying leave-one-out-cross-validation, these models' predictive accuracies were $69 \%$ accuracy for unindicative and $79 \%$ accuracy for indicative items. A permutation test on the predicted outcomes confirmed that this prediction is significantly better than guessing their assignment in both cases $(p=0.001$ and $p<0.001$ respectively). However, these predictions are only moderately better than the base rate, i.e. the accuracies achievable if predicting all subjects to reach perfect performance for unindicative items (54\%) and no subject to reach perfect performance for indicative items $(63 \%)$.

We then performed the same analysis for predicting whether subjects would display zero accuracy on indicative items only (32\% of subjects), since, unsurprisingly 
given the post-hoc grouping of subjects, none of the subjects displayed zero accuracy on unindicative items during the unsupervised phase. The logistic regression model revealed that the probability of achieving zero accuracy significantly decreased with initial performance $\left(\beta_{a c c}=-3.4\right.$ with $\left.p=0.001\right)$. Applying leave-one-out-crossvalidation, this models' predictive accuracy was $69 \%$ which a permutation test confirmed to be significantly better than guessing $(p=0.03)$, but only marginally better than prediction based on the base rate $(68 \%)$.

In summary, these results are consistent with our original predictions. On average, subjects who had high initial accuracy were more likely to improve without supervision while those with low initial accuracy were more likely to worsen. However, these results are conditioned on the subset of subjects that displayed either conformant or non-conformant performance by the end of the experiment, whereas nearly half of the subjects tested did not resemble either of these two categorisation behaviours. To ensure our results still hold when including these other subjects, we now turn to analysing all the data.

\subsubsection{All semi-supervised subjects.}

First, we tested whether we could still predict the post-hoc grouping into conformant, non-conformant and other subjects from their initial supervised performance, now looking at the entire population. As before, we performed the same leaveone-out-cross-validation analysis, now employing a multinomial logistic regression model to predict all three group outcomes which yielded a predictive accuracy of $66 \%$ which again was significantly better than guessing $(p<0.001)$.

Next, we sought to predict performance change. While we observed ceiling effects for the non-conformant and conformant subjects, the remaining group of subjects showed evidence for regression to chance performance (see supplementary material). Minimising the impact of these effects, we again predicted whether subjects would reach perfect or zero accuracy on at least one unsupervised block from their performance on block 3, now for the entire data. We observed that $29 \%$ and $26 \%$ of subjects reached perfection on unindicative and indicative items respectively. We find that again the probability of reaching perfect accuracy significantly increased with initial performance $\left(\beta_{a c c}>3.5\right.$ with $p<0.001$ for both item types). Applying leave-one-out-cross-validation, the predictive accuracy for unindicative items was $77 \%$ which confirmed to be above chance $(p<0.001)$ but only marginally higher than the base rate $(71 \%)$. However, predictive accuracy for indicative items did not significantly exceed the base rate $(74 \%$ for indicative items; $p=0.35)$. Applying the same analysis for reaching zero performance observed in $21 \%$ of subjects (again only for indicative items), we find that the probability of this significantly decreased with initial performance $\left(\beta_{a c c}=-2.8\right.$ with $p=0.002$ for indicative items). Again, predictive performance of the model did not exceed the base rate of $79 \%(p=1)$.

A closer look at subjects' responses revealed that some of those who did not pass either of the two performance criteria did recover at least a subset of stimulus 
groups but assigned them to the wrong categories. That is, even though these subjects appear to have attended to the right dimension, and made consistent choices, those choices were consistently incorrect. In addition, a small proportion of subjects attended to the task-irrelevant dimension but reversed the key mapping during the unsupervised phase showing as good performance on indicative items and poor performance on unindicative items. Since predicting changes in accuracy for these subjects does not fully reflect their underlying learning, we sought to confirm that the degree to which subjects improve relative to the task they might have thought was correct, depends on how well they are performing on that task before feedback withdrawal. We tested this by predicting whether subjects would achieve perfect consistency in their responses on at least one of the unsupervised blocks. We measured subjects' consistency for each block as the proportion of identical responses to each of the four stimulus groups (the average of this we call cluster consistency) and as the proportion of identical responses to the body dimension on the $50 \%$ most extreme body shapes (the average of this we call body consistency). $19 \%$ and $41 \%$ of subjects reached perfect cluster and body consistency respectively. A logistic regression model revealed that the probability of achieving perfect consistency on at least one unsupervised block increased with consistency on block 3 ( $\beta_{\text {cons }}=8.3$ with $p<0.001$ for cluster consistency and $\beta_{\text {cons }}=3.6$ with $p=0.002$ for body consistency). Predictive accuracy for reaching perfect cluster consistency was $80 \%$ which was not significantly better than guessing $(p=0.24)$ and in fact did not exceed base rate performance $(81 \%)$. Predictive accuracy for reaching perfect body consistency was $65 \%$ which was significantly better than guessing $(p=0.001)$ and moderately above base rate performance (59\%).

In summary, these results echo the findings reported for the conformant and non-conformant subjects and thus support our predictions. While we were able to predict whether subjects' would pass the ultimate conformant or non-conformant categorisation criterion from their initial performance, prediction of performance change was not possible when including the additional subjects due to the overlap in initial accuracies between the three subject groups and their different behavioural patterns (improvement and worsening versus regression to chance). 